\title{
Target optimization studies for surface muon production
}

\author{
Adriana Bungau, ${ }^{*}$ Robert Cywinski, and Cristian Bungau \\ University of Huddersfield, School of Applied Sciences, Huddersfield HD1 3DH, United Kingdom \\ Philip King, and James S. Lord \\ STFC-Rutherford Laboratory, ISIS Neutron and Muon Source, Didcot OX11 OQX, United Kingdom
}

(Received 19 July 2013; published 20 March 2014)

\begin{abstract}
The current paper discusses possible designs for a stand alone muon target for MuSR studies of condensed matter science. Considering the ISIS $7 \mathrm{~mm}$ graphite target as a reference, GEANT4 simulations have been performed in order to optimize the target parameters with respect to muon and pion yield. Previous studies suggested that the muon production can be optimized by using a thin graphite slab target with an incident proton energy significantly lower than initially considered. Surface muon production obtained by firing an $800 \mathrm{MeV}$ proton beam energy onto the target is simulated and potential improvements to the target material, geometry and angle orientation with respect to the incoming proton beam as well as an estimated performance of the muon target are presented in this paper. Implications for the ISIS muon facility are also discussed. A comparison of the pion production cross section between experimental data and three theoretical models for the latest four GEANT4 versions is also included in this paper.
\end{abstract}

DOI: 10.1103/PhysRevSTAB.17.034701

PACS numbers: 29.25.-t

\section{INTRODUCTION}

Intense beams of polarized muons are required by the MuSR technique to probe deep inside materials in order to tackle fundamental problems in condensed matter science, chemistry, medicine, and particle physics [1-4]. The MuSR technique uses the muon's spin to examine the structural and dynamical processes in bulk material on atomic and subatomic scale. Muons are implanted into material samples and their spins precess around the local atomic and nuclear magnetic fields in the material. The unstable muons decay with an average lifetime of 2.2 microseconds into a positron and because the positrons are emitted preferentially in the direction of the muons' spin, the precessional motion or the depolarization of the muon spins by the internal magnetic fields can be determined by observing the angular and time dependent distribution of emitted positrons. Whilst MuSR at the current muon facilities has already made a major contribution to our understanding of phenomena as diverse as superconductivity [5,6], itinerant magnetism [7], spin glasses [8], semiconductors [9] etc., it is clear that much more could be learned if more intense muon beams were routinely available to enable, for example, rapid parametric investigations as functions of magnetic field, temperature, pressure, or sample composition [10].

\footnotetext{
*A.Bungau@hud.ac.uk

Published by the American Physical Society under the terms of the Creative Commons Attribution 3.0 License. Further distribution of this work must maintain attribution to the author(s) and the published article's title, journal citation, and DOI.
}

MuSR experiments generally (though not always) rely upon polarized beams of relatively low energy positive muons which rapidly thermalize within a few $\mathrm{mm}$ of the sample being studied. Fortunately such polarized muons, known as surface muons with momenta of $\approx 28 \mathrm{MeV} / c$, are produced by the decay of positive pions which are at rest at the surface of the production target. The polarized surface muon beams are then guided with magnetic fields to the muon spectrometers.

The MuSR experiments are carried out at the continuous muon beam facilities at PSI (Switzerland) [11] and TRIUMF (Canada) [12] and the pulsed beam facilities at ISIS (U.K.) [13] and J-PARC (Japan) [14]. The high cost related to accelerator construction and operation have resulted in the so-called multipurpose facilities where muon and neutron experiments are carried out all together, providing complementary information in a wide variety of science. In this respect the design of each of the muon facilities, and in particular the geometry and efficiency of the pion/muon target, is essentially a compromise which leads to a suboptimal muon delivery rate to the MuSR spectrometers [15].

A stand alone muon facility would be desirable for muon experiments and it is of technical interest to consider how muon production can be optimized. Therefore using the ISIS target as a reference simulations have been performed to provide an optimized solution for the target design with respect to the pion/muon production. Implications of the optimized solutions for our U.K. facility are also discussed in this paper. 

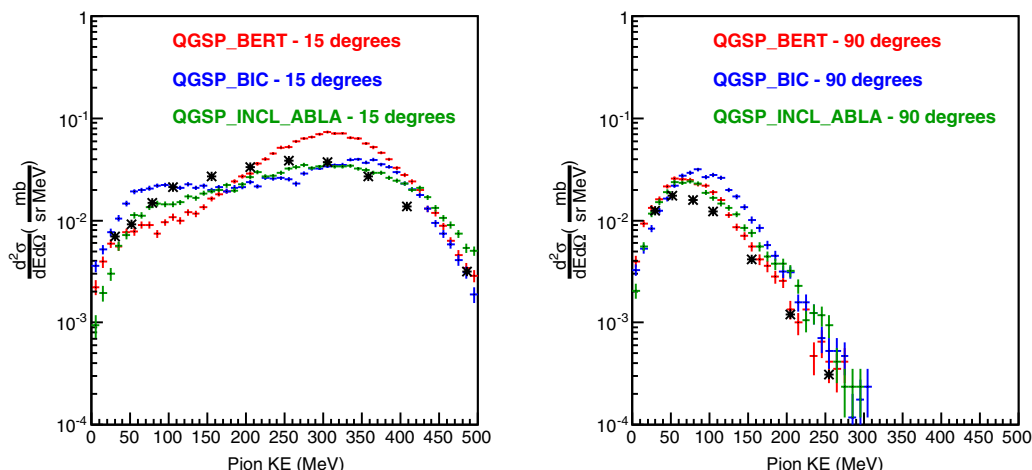

(a)
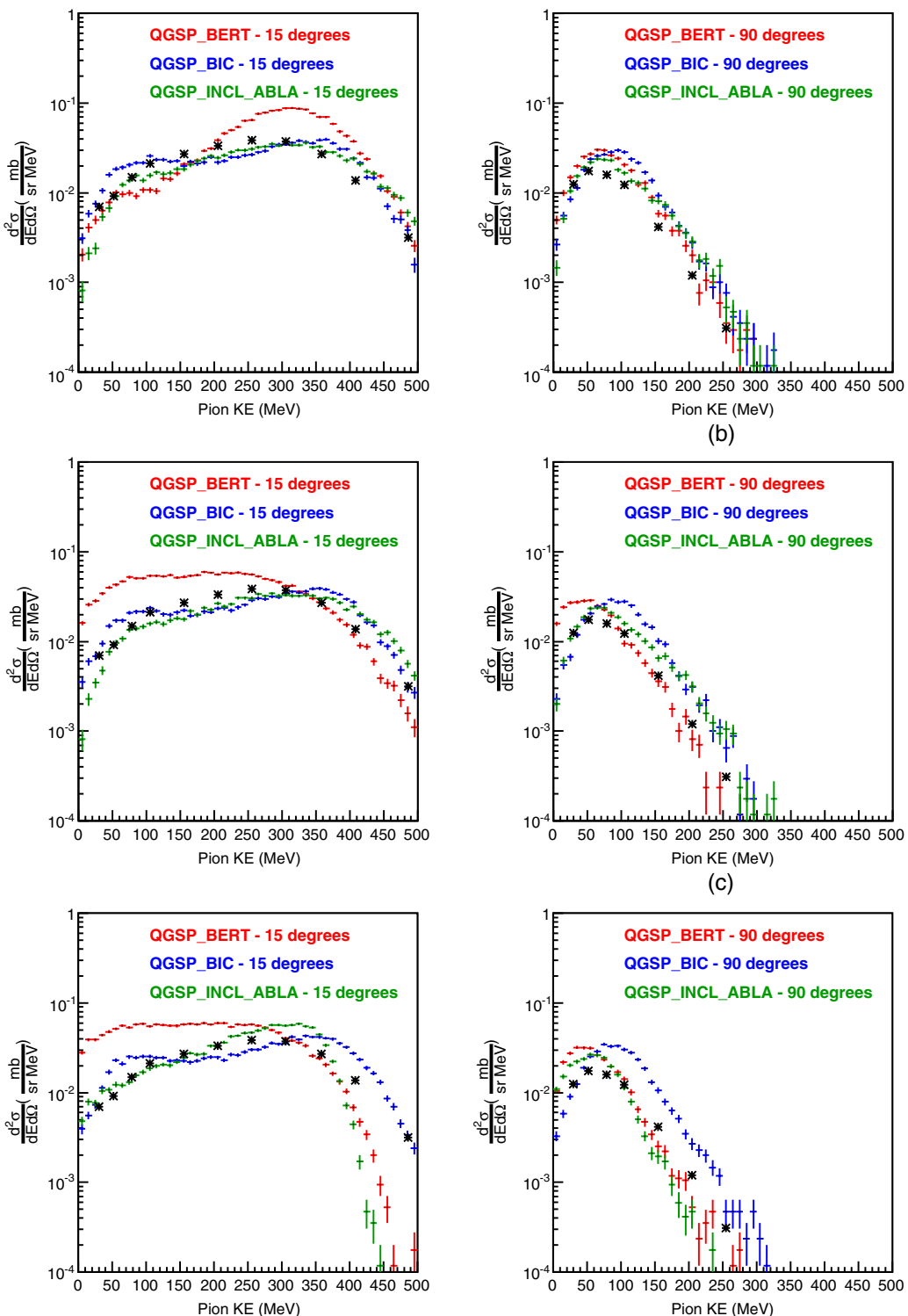

(b)

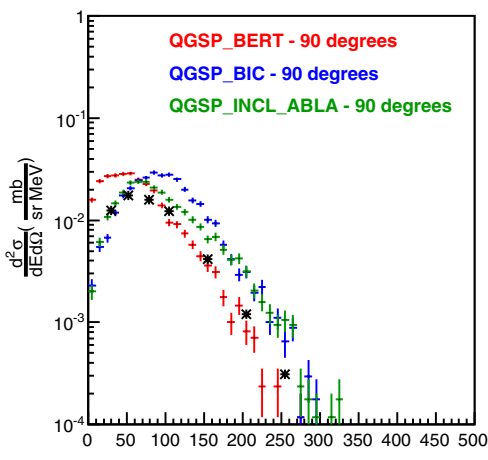

(c)

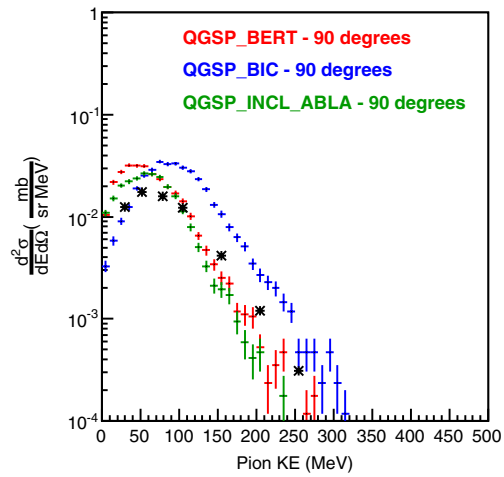

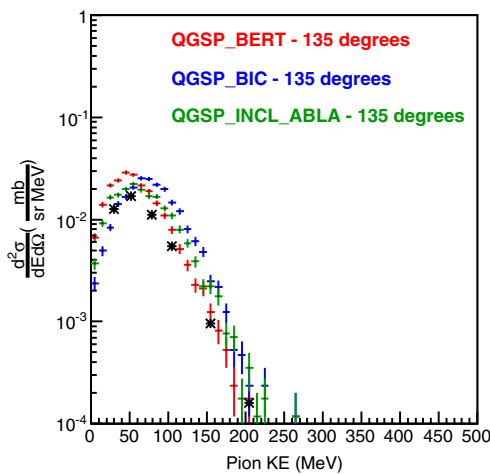

(d)
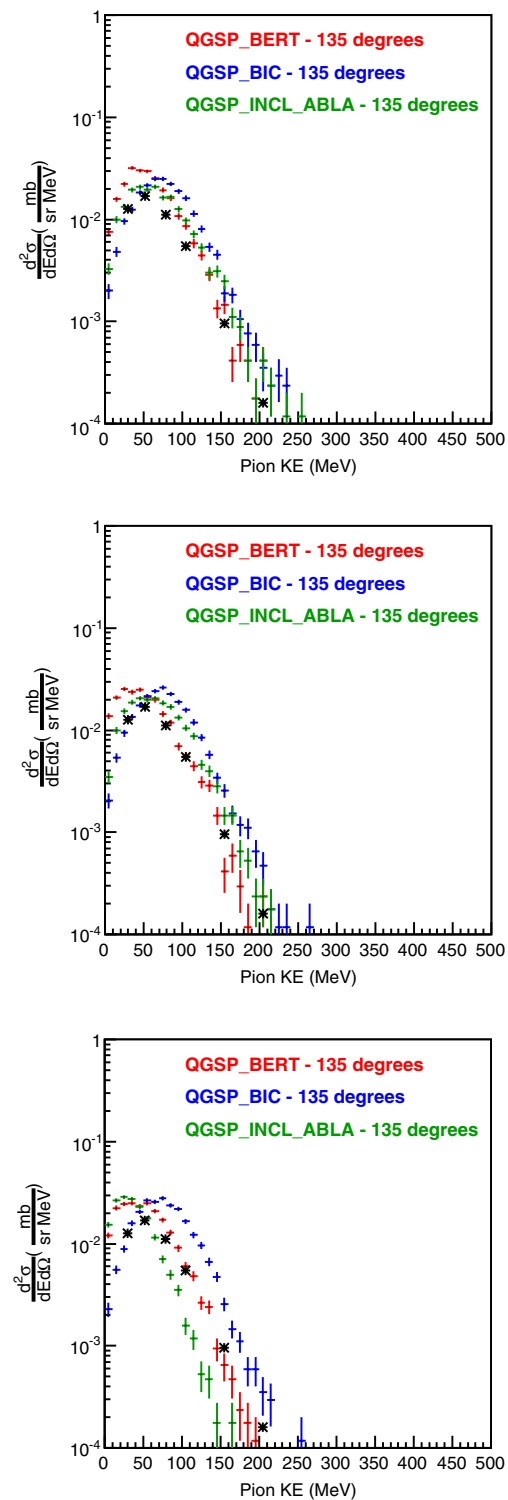

FIG. 1. Double differential cross-section predictions with the last four GEANT4 versions for positive pion production. Three physics packages (QGSP-BERT, QGSP-BIC, QGSP-INCL-ABLA) are compared with experimental data. (a) Pion production double differential cross section using the GEANT4 version geant4.9.3.p02. (b) Pion production double differential cross section using the GEANT4 version geant4.9.4.p04. (c) Pion production double differential cross section using the GEANT4 version geant4.9.5.p02. (d) Pion production double differential cross section using the GEANT4 version geant4.9.6.p01. 


\section{THE SIMULATION CODE}

Optimization studies have been performed using the Monte Carlo code GEANT4 [16] which simulates particle interactions and transport through the target material. Validation studies for pion production differential cross section for $730 \mathrm{MeV}$ proton energy were performed using three physics packages QGSP-BERT [17], QGSP-BIC [18], and QGSP-INCL-ABLA [19] and the results are compared with the experimental data. The data were measured by Cochran et al. [22] at the Lawrence Radiation Laboratory for different target materials and detector angles. Figure 1 shows the double differential cross section for positive pion production at 15, 90, and 135 degrees with respect to the proton beam.

The validation results have shown a good agreement between simulation and experimental data for version geant4.9.3.p02 [20], however for later releases (versions geant4.9.4.p04, geant4.9.5.p02, and geant4.9.6.p01) the results are noticeably different. In these releases the function getMomModuleFor2toMany from the class G4ElementaryParticleCollider has been modified by eliminating a special case initialization and thus obtaining a better agreement with data for pion and kaon interactions with hadrons at the higher end of the kinematic range. However, this modification significantly changed the comparison of Bertini model output to data for hadron-nucleon cascades [21].

At small angles the previous versions geant4.9.3.p02 and geant4.9.4.p04 seem to be in reasonable good agreement with the experimental data for all three cascade models. Versions geant4.9.5.p02 and geant4.9.6.p01 however show significant discrepancies especially for Bertini and binary cascade models. At large angles the QGSP-BERT gives a better agreement with data while the QGSP-BIC tends to overestimate the pion production. The QGSP-INCL-ABLA also overestimates the data apart from version geant4.9.6.p01 where the experimental results are underestimated. Therefore, in our simulations we used the version geant4.9.3.p02 with the QGSP-BERT package as it is more likely to produce sensible results for our particular case. This package comprises several physics models:

(i) the Bertini cascade model (BERT) for intranuclear cascade followed by preequilibrium and evaporation phases of the residual nucleus for proton, neutron, pion, and kaon interactions below $9.9 \mathrm{GeV}$;

(ii) parametrized models for all remaining hadrons;

(iii) parametrized capture and fission for low energy neutrons;

(iv) hadronic elastic scattering;

(v) standard electromagnetic physics;

(vi) chiral invariant phase space (CHIPS) model of nuclear capture of negatively charged particles at rest;

(vii) parametrized muon-nuclear interactions; (viii) CHIPS model for gamma-nuclear and electronnuclear interactions;

(ix) quark-gluon string model for all hadronic interactions above $12 \mathrm{GeV}$ followed by the precompound model for preequilibrium and evaporation phases of the residual nucleus;

(x) low energy parametrized model for hadronic interactions between $9.5-25 \mathrm{GeV}$;

(xi) quasielastic scattering.

\section{RESULTS}

Because all but one of the current muon facilities (TRIUMF is the exception) coexist symbiotically with the neutron facilities and the muon targets are placed upstream of the neutron targets, the proton transmission through the muon targets and a following set of collimators is an important factor that must be taken into account when designing the targets. In general, the total beam loss induced by the muon production targets should not exceed $10 \%$. At ISIS for example the proton transmission is around $96 \%$ while at J-PARC the beam loss at the $20 \mathrm{~mm}$ muon target is $6.5 \%$ [23]. Such restrictions would not be necessary in a stand alone muon facility.

Simulation studies have shown the low rates of muons that are currently used in muon experiments. For the $7 \mathrm{~mm}$ graphite target used currently at the ISIS muon facility the proton transmission is $96.77 \%$. From these $96.77 \%$ transmitted protons, $69.58 \%$ pass through the target without interacting while $27.19 \%$ of the protons reach the downstream neutron target interacting in the muon target via other processes (multiple scattering, elastic scattering, ionization, etc.) From the remaining 3.23\% nontransmitted protons which also interact in the target only $2.04 \%$ interact inelastically and $1.19 \%$ interact via other processes. From the $2.04 \%$ protons only $0.56 \%$ will produce pions.

Substantial gain in intensity can be achieved through optimization of the target material. At current muon facilities low-Z materials like graphite and beryllium are chosen for the target as both have a low density such that the proton beam passes through the target without significant interactions. Also both materials have a high melting point ( $3800 \mathrm{~K}$ for graphite and $1560 \mathrm{~K}$ for beryllium) as the target is expected to run hot in vacuum. Beryllium in particular has also a high temperature stability and a low coefficient of expansion with temperature. For a stand alone muon target, nickel can be considered as a potential candidate due to the high melting point $(1726 \mathrm{~K})$ and stress resistance.

A Gaussian beam of $10^{9}$ protons with a radius of $10 \mathrm{~mm}$ was used in all simulations. The target was surrounded in our simulations by a spherical shell detector with an inner radius of $13 \mathrm{~cm}$ and an outer radius of $14 \mathrm{~cm}$. The spherical shell was made of vacuum to avoid particle scattering. The variation of the total and surface muon yield as a function of target thickness is shown in Fig. 2(a). All muons having the 


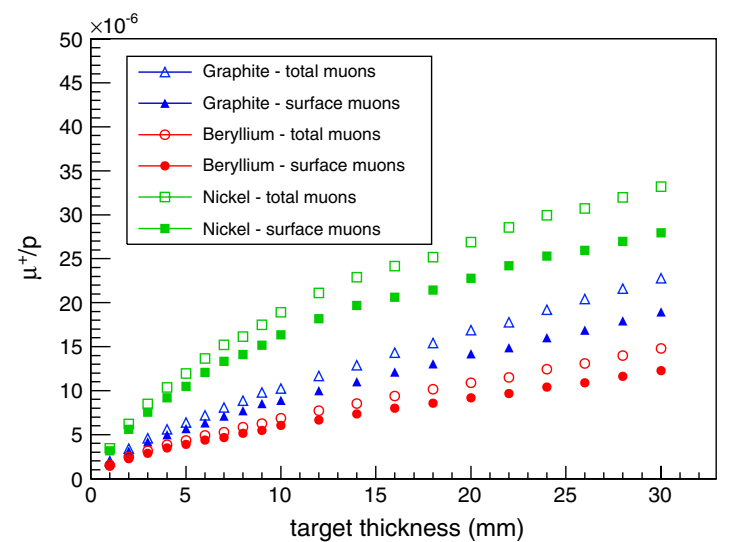

(a)

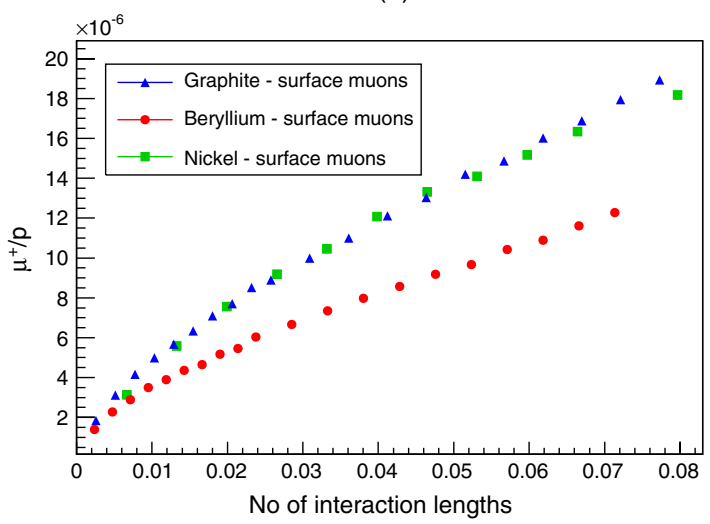

(b)

FIG. 2. The comparison of the muon yield per proton for three different materials. (a) Variation of the muon yield with the target thickness. (b) The muon yield as a function of the interaction length.

momentum in the range $0-100 \mathrm{MeV} / c$ are recorded in the shell. However, if a cut is applied in practice at $30 \mathrm{MeV} / c$, the surface muons produced by pions decaying at rest near the target surface with sufficient energy to escape from inside the target will be detected together with background muons (muons coming from pions in flight and having a momentum lower than $30 \mathrm{MeV} / c$ ). In a stand alone muon facility a nickel target would give a substantial higher muon yield than graphite and beryllium especially for thicker targets. For example, a $30 \mathrm{~mm}$ Ni target would produce 4 times more surface muons than a $7 \mathrm{~mm}$ graphite target.

In addition to this comparison between the three materials, the target thickness can be expressed in terms of the number of proton interaction length and the corresponding muon yields can be compared for the same number of interaction lengths in the three materials. The muon yield given by the same number of proton inelastic interactions in these materials enables us to compare the muon yields per interaction rather than target thickness. From Fig. 2(b) it can be seen that the muon yield per proton interaction is lower in Be than in graphite and Ni. An in-depth study of different target configurations addressing all the engineering aspects of each solution must be done further.
Because the surface muon production at small thicknesses is nonlinear, the muon yield can be increased even further if the $7 \mathrm{~mm}$ graphite target is split along the proton beam line into two or three sets of slabs such that the total thickness is still $7 \mathrm{~mm}$. Thus more pions will be decaying at rest at the surface of the slabs producing even more surface muons. The distance between the slabs is varied gradually and the surface muons detected by the shell as a function of slab distance is presented in Fig. 3.

If the slices are too close, a fraction of the surface muons does not reach the shell because of channeling between them. Therefore the surface muon yields for two and three slabs are similar when the separation distance is below $20 \mathrm{~cm}$. As the distance is increased the muon production rate is going up and then flattens as all the muons produced in the slabs reach the detector. For a twoslab design geometry the surface muon production increases with $21 \%$ compared with the initial design. For the set of three slabs, the muon production increases with $40 \%$.

The thickness of the set of slabs was kept constant in the previous simulations. However, by varying the orientation angle, different slab thicknesses will be presented in the beam path leading to more proton interactions inside the target and a higher surface muon yield. Figure 4 shows the variation of surface muon yield with the separation between the slabs and the slab angle orientation for the two- and three-slabs design case. At small separation distances the muon yield is higher at smaller angles because a higher total slab thickness is presented in the proton beam path. At large separation distances a higher muon yield is obtained for larger angles because muons that are produced on both sides of each of the slabs are now detected.

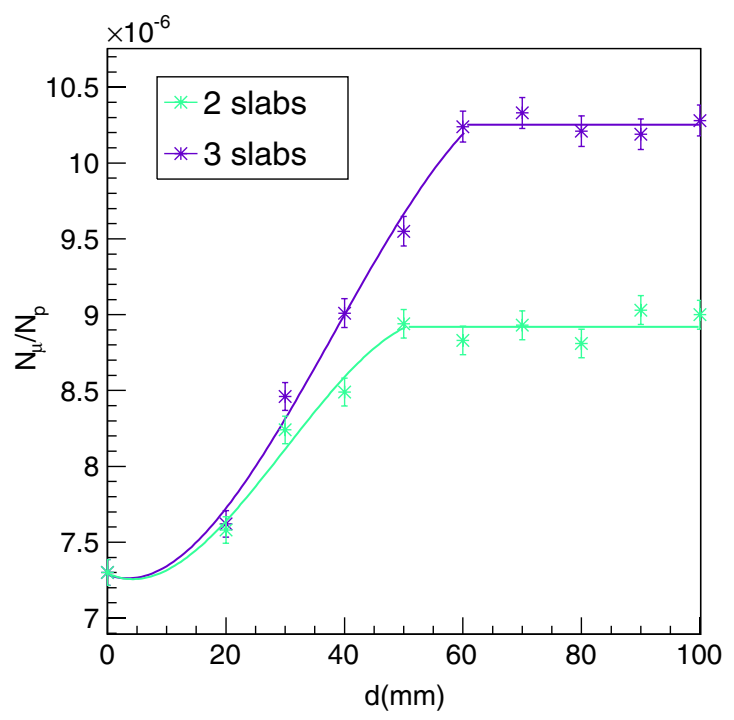

FIG. 3. Variation of the surface muon yield with the slab separation distance. The muons are detected by the spherical shell. 


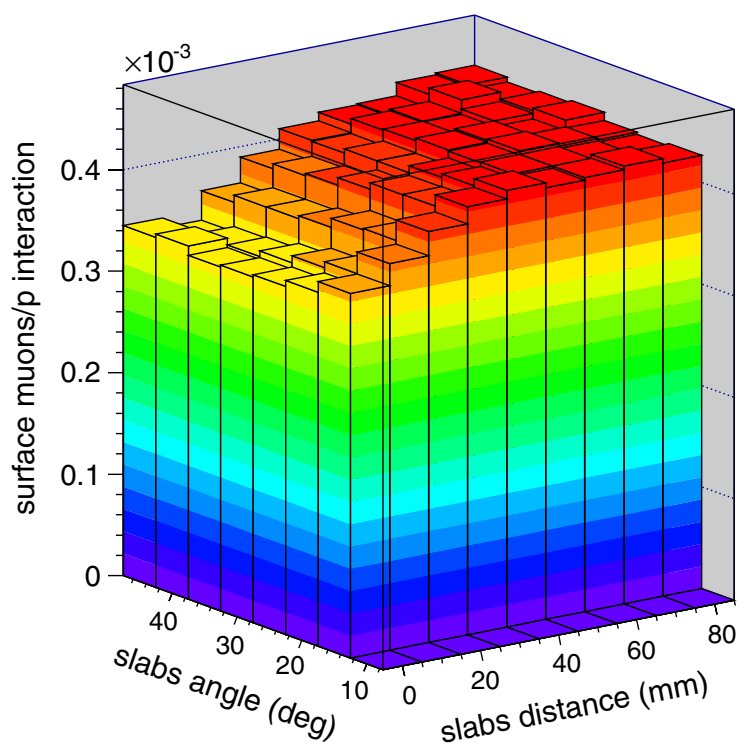

(a)

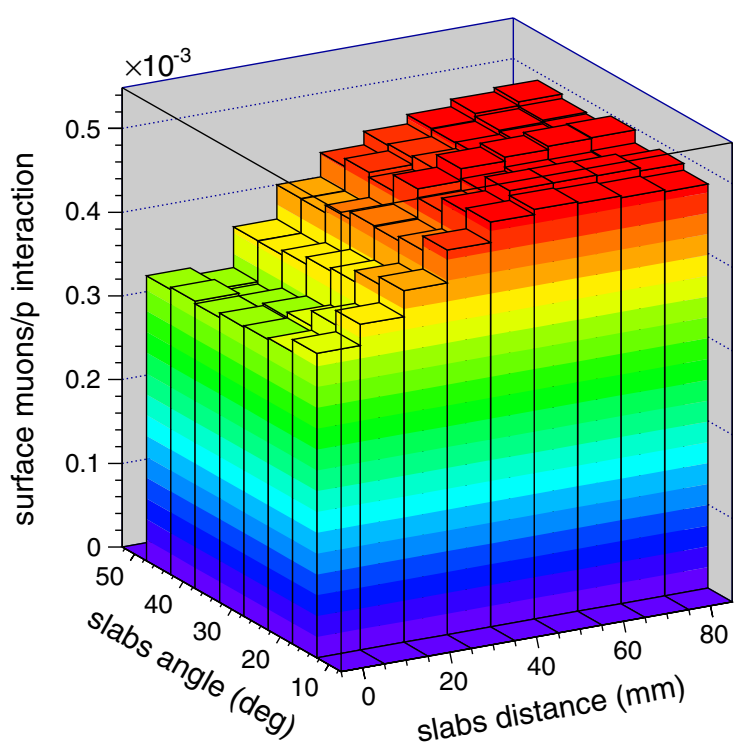

(b)

FIG. 4. Variation of the surface muon yield with distance between the slabs and slab angle orientation. (a) The two-slabs design case. (b) The three-slabs design case.

\section{IMPLICATIONS FOR ISIS}

The optimized solutions described previously have been implemented in the simulations of the ISIS muon target. The pulsed muon facility at ISIS facility has operated successfully for many years serving a wide international MuSR community. The ion source of the ISIS accelerator injects negatively charged hydrogen ions into a linac which accelerates and transports the ions to the synchrotron where, on injection, they are stripped of their electrons by a thin foil, leaving bare protons. The synchrotron then accelerates the protons to $800 \mathrm{MeV}$. The resulting extracted proton beam has a double pulse structure with $2.5 \times 10^{13}$ protons per double pulse, with a frequency of $50 \mathrm{~Hz}$, resulting in a nominal proton beam current of $200 \mu \mathrm{A}$.

The extracted proton beam then passes through a thin graphite muon production target with dimensions $50 \times 50 \times 7 \mathrm{~mm}$, oriented at 45 degrees to the proton beam and giving an effective length of $10 \mathrm{~mm}$ along the beam (Fig. 5). The interaction of the proton beam with the target nuclei produces pions which decay into muons. The primary requirement for the target is to produce a large number of pions and hence muons in order to achieve acceptable intensities for physics experiments.

Low-Z target materials are preferred in order to maximize the pion production and to minimize both the rate of absorption of secondary pions and the multiple scattering of the proton beam itself within the target material. Second, the target must survive in the extreme condition of an intense high energy, pulsed proton beam by dissipating the energy deposition and by surviving both the pressure waves induced by beam pulses and also the long-term effects of radiation damage.

The surface muons so produced are extracted into two beam lines each at 90 degrees with respect to the proton beam and these two beam lines are separated from the main proton beam and target vacuum vessel by a thin aluminum window. The beam window has a diameter of $8 \mathrm{~cm}$ and is situated at $15 \mathrm{~cm}$ from one side of the target. The production target is followed by a set of two collimators which are angled cones of $40 \mathrm{~cm}$ length and are made of $\mathrm{Cu}$. The first collimator has an inner radius of $37.5 \mathrm{~mm}$ and an outer radius of $54.15 \mathrm{~mm}$ and the second collimator has an inner radius of $51 \mathrm{~mm}$ and an outer radius of $61.4 \mathrm{~mm}$.

The transmitted proton beam goes on to impact the tungsten neutron production target situated $20 \mathrm{~m}$ downstream. Because the muon facility is essentially parasitic with respect to the neutron facility the proton transmission through the muon production target, defined as the fraction

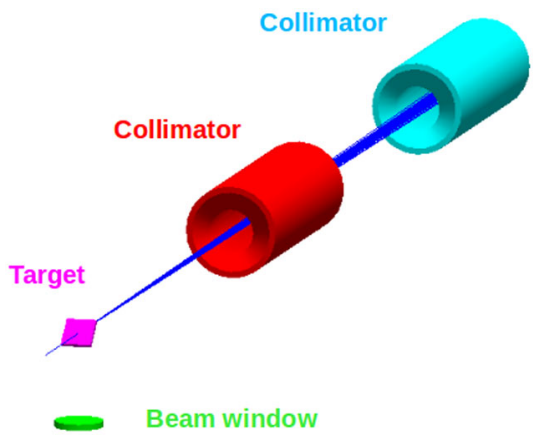

FIG. 5. GEANT4 modeling of the ISIS muon target, beam window, and collimators. The target is tilted at 45 degrees and the muons produced in the target are collected by the beam window. The transmitted proton beam passes through the collimation system and impacts further on the neutron target situated $20 \mathrm{~m}$ downstream. 
TABLE I. Total and surface muon yield detected by the spherical shell for a similar proton transmission.

\begin{tabular}{lccc}
\hline \hline Material & $\begin{array}{c}\text { Thickness } \\
(\mathrm{mm})\end{array}$ & $(\mu / p) \times 10^{-6}$ & $\begin{array}{c}(\text { Surface } \mu / p) \\
\times 10^{-6}\end{array}$ \\
\hline Graphite & 7 & $8.07 \pm 0.09$ & $7.09 \pm 0.08$ \\
$\mathrm{Be}$ & 7 & $5.29 \pm 0.07$ & $4.65 \pm 0.06$ \\
$\mathrm{Ni}$ & 1.6 & $5.22 \pm 0.07$ & $4.71 \pm 0.07$ \\
$\mathrm{Be}$, Ni coating & $(6+1)$ & $6.62 \pm 0.08$ & $5.84 \pm 0.08$ \\
\hline \hline
\end{tabular}

of protons passing through the collimation system, must be maintained at a predetermined level to prevent loss in neutron intensity at the neutron instruments, thus limiting the muon production rates.

The requirement for $96 \%$ proton transmission is satisfied by either a $7 \mathrm{~mm}$ graphite or beryllium target or a $1.6 \mathrm{~mm}$ nickel target. Figure 2 showed that $7 \mathrm{~mm}$ graphite gives a higher surface muon yield than either $1.6 \mathrm{~mm} \mathrm{Ni}$ or $7 \mathrm{~mm}$ Be. However, we have investigated the potential optimization of a Be target in more detail. Beryllium can potentially evaporate or sputter from the target surface in the intense proton beam, thereby contaminating the beam line and creating a health hazard. Nickel has been shown to be a suitable coating material for conventional low-Z targets. Clearly the $\mathrm{Ni}$ coating should be sufficiently uniform and robust to prevent sputtering and evaporation of $\mathrm{Be}$, but at the same time be sufficiently thin to not compromise the proton transmission through the composite target. We find that a Be target of $6 \mathrm{~mm}$ thickness coated with $0.5 \mathrm{~mm} \mathrm{Ni}$ layer on all sides gives a proton transmission of $95.01 \%$. The total and surface muon yield given by graphite, $\mathrm{Be}, \mathrm{Ni}$, and $\mathrm{Be} / \mathrm{Ni}$ targets for a comparable proton transmission are presented in Table I.

The distribution of surface muon production from the composite target is shown in Fig. 6, in which different colors represent the contribution of the two materials to the

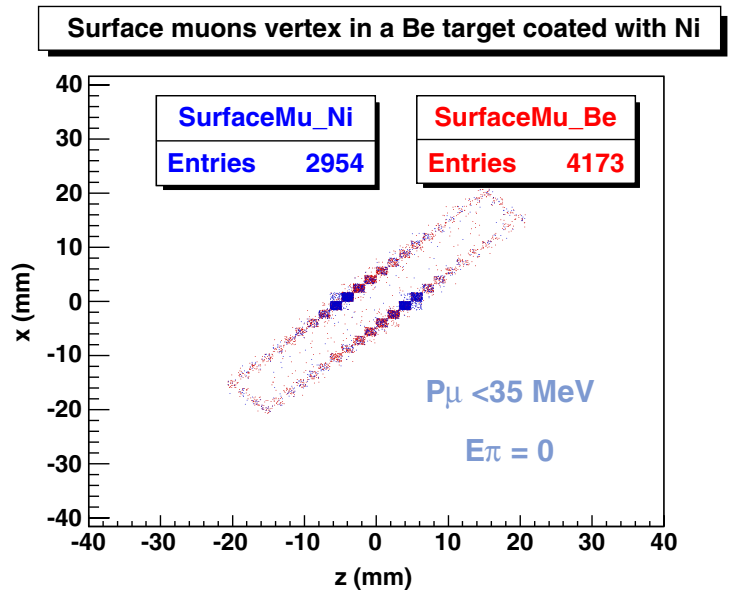

FIG. 6. Contribution of both materials to the surface muon production. Different colors represent the material in which the parent pion was produced.
TABLE II. Total and surface muon yield detected by the ISIS beam window for similar proton transmissions.

\begin{tabular}{lccc}
\hline \hline Material & Thickness $(\mathrm{mm})$ & $(\mu / p) \times 10^{-6}$ & $\begin{array}{c}(\text { Surface } \mu / p) \\
\times 10^{-6}\end{array}$ \\
\hline Graphite & 7 & $0.18 \pm 0.01$ & $0.16 \pm 0.01$ \\
Be & 7 & $0.13 \pm 0.01$ & $0.11 \pm 0.01$ \\
$\mathrm{Ni}$ & 1.6 & $0.11 \pm 0.01$ & $0.10 \pm 0.01$ \\
$\mathrm{Be}, \mathrm{Ni}$ coating & $(6+1)$ & $0.15 \pm 0.01$ & $0.13 \pm 0.01$ \\
\hline \hline
\end{tabular}

pion production reflecting the material in which the pions are produced, irrespective of the material in which the pion decays. The contribution of beryllium to the surface muon production is $59 \%$ while for nickel it is $41 \%$.

The performance of the plain and coated targets with respect to those muons detected by the ISIS beam window is presented in Table II.

It can be seen that the $7 \mathrm{~mm}$ graphite target has the optimum performance of all four targets. The validity of the results obtained previously with the spherical shell configuration relies on the fact that the surface muon production is isotropic [20]. Thus the ISIS beam window is capturing $\sim 2 \%$ of the total number of surface muons produced in the target. This result is to be expected since the solid angle to the beam window is $\Omega=0.071 \pi$ and the surface muon production is isotropic.

However, only those muons emerging from the target within a vertical acceptance of $\pm 5 \mathrm{~mm}$ and a horizontal acceptance of $\pm 30 \mathrm{~mm}$, with divergence of $35 \mathrm{mrad}$ in the horizontal direction and $180 \mathrm{mrad}$ in the vertical direction and momentum in the range $25-27 \mathrm{MeV} / c$ per unit charge are accepted by the muon beam line. The muon beam is fully polarized and this polarization is maintained as the

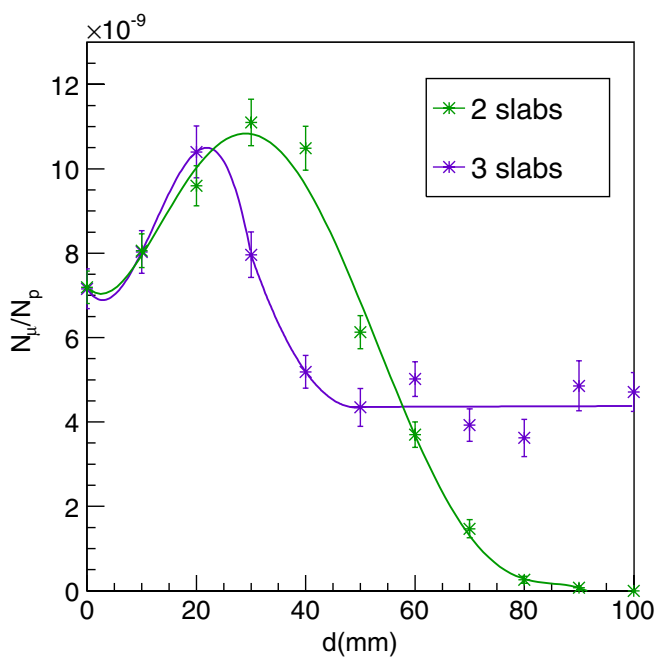

FIG. 7. Variation of the surface muon yield as a function of slabs distance for a 45 degrees angle orientation. The muons are detected by the ISIS beam window and the appropriate selection cuts are applied. 
beam is transported to the muon spectrometers. For the $7 \mathrm{~mm}$ graphite target used at ISIS, $\approx 7 \times 10^{-9}$ surface muons per proton are detected by the beam window.

Given that graphite appears to be the most efficient target material, we have therefore explored the effect of multiple graphite slab geometry at the ISIS muon production target. For this particular case the muons entering at large angles are excluded because they will not enter the first quadrupole and therefore will not be transmitted by the muon beam line. In order to estimate the usable muon yield, we have applied the acceptance and angular cuts appropriate to

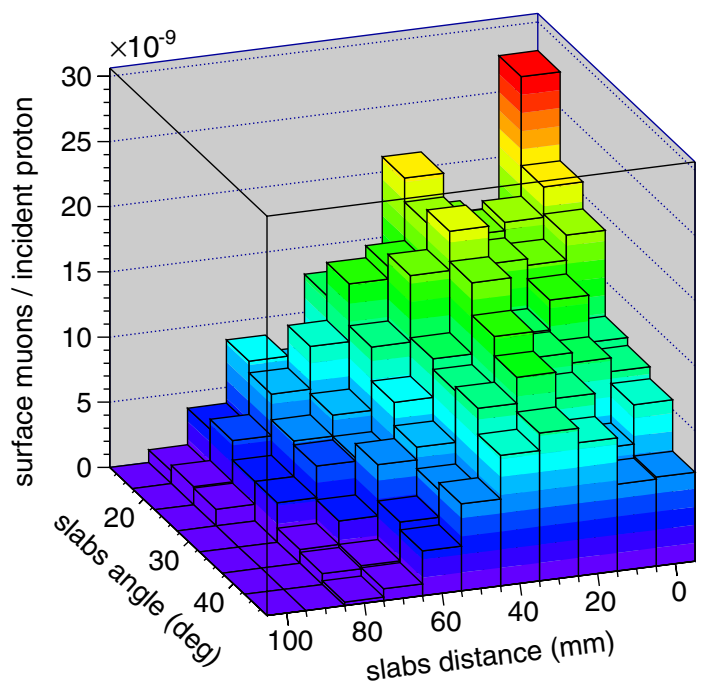

(a)

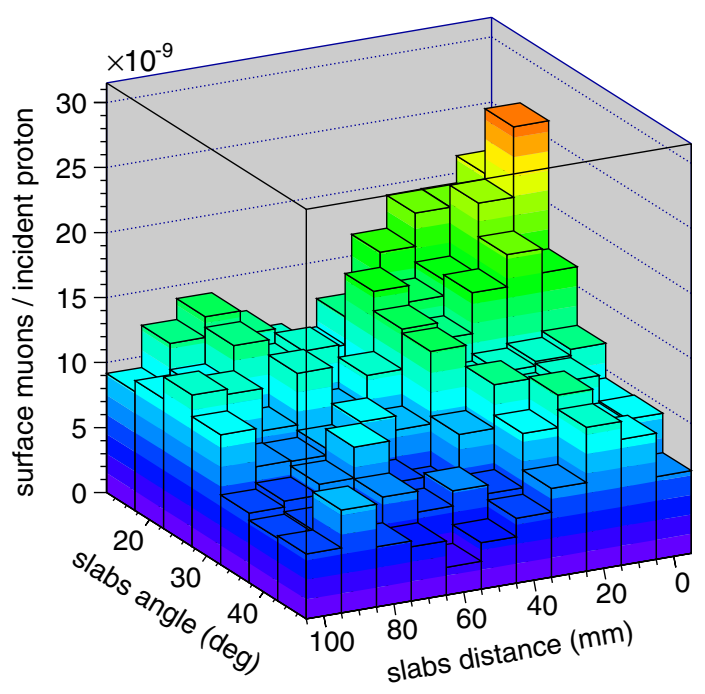

(b)

FIG. 8. Variation of the surface muon yield detected by the ISIS beam window with distance between the slabs and slab angle orientation. Acceptance and angular cuts are applied in both cases. (a) Variation of surface muon yield with slab distance and angle for the two-slabs design case. (b) Variation of surface muon yield with slab distance and angle for the three-slabs design case. the ISIS muon beam line. The muons which do not pass the above selection cuts cannot be used in a MuSR experiment. Figure 7 shows the results.

For a two-slab geometry there is an increase in the muon production rate but after $\sim 30 \mathrm{~mm}$, which seems to be the optimum distance, the rate decreases as the resulting muons are no longer captured by the $8 \mathrm{~cm}$ diameter beam window. For a three-slab geometry the rate increases initially with separation being optimum at $20 \mathrm{~mm}$ separation distance but when the distance between the slabs is greater than $\sim 50 \mathrm{~mm}$, the only surface muons collected by the beam window are those produced in the central slab. Having a set of two slabs results in a higher surface muon yield of 54\% with respect to the present target design configuration, for the optimum distance of $30 \mathrm{~mm}$ between the slabs. The set of three slabs results in an increase in the surface muon yield of 50\% with respect to the present target design

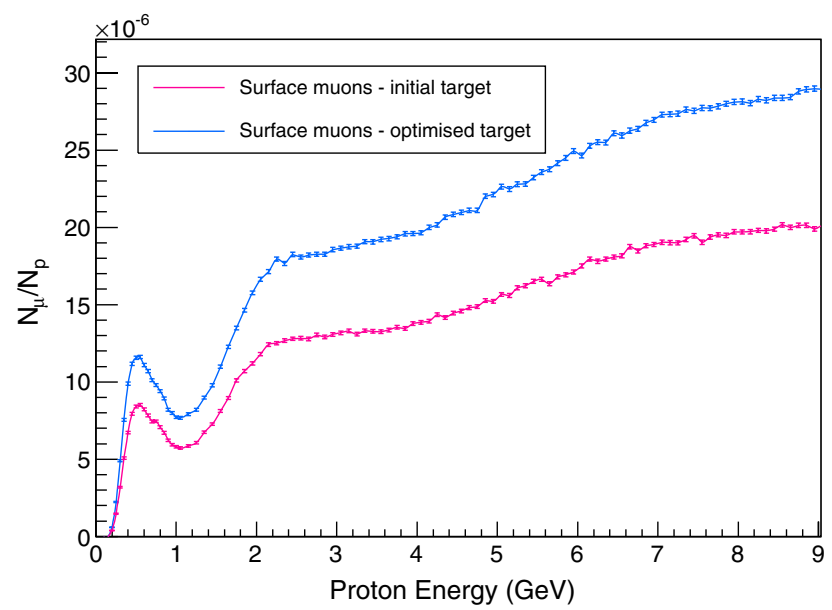

(a)

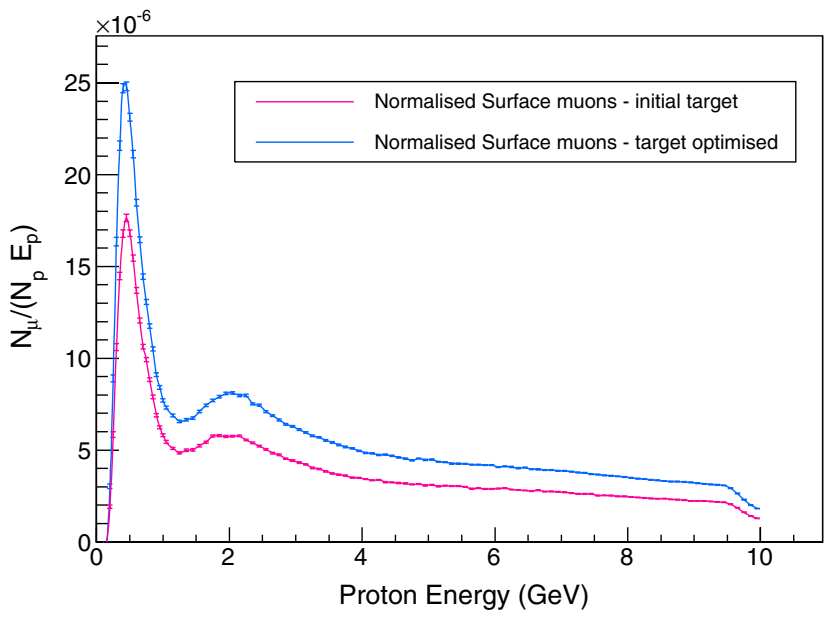

(b)

FIG. 9. Variation of surface muon yield with proton energy for the current ISIS target parameters and the optimized slab target design. (a) Muon multiplicity. (b) Muon yield per unit energy of incident proton. 
configuration, for the optimum distance of $20 \mathrm{~mm}$ between the slabs.

Since the total thickness of the slabs is always equal to the thickness of the original target, the proton transmission does not depend on the number of slabs or on the distance between them, it only depends on the angle of orientation to the proton beam. For the two and three design case and 45 degrees angle orientation, the proton transmission is $96.89 \%$ and $97 \%$, respectively.

The variation of surface muons with the slab separation distance and the angular orientation after applying the acceptance and angular cuts for the ISIS beam window is shown in Fig. 8. Taking into account that the orientation angle must be larger than 35 degrees in order to maintain the proton transmission above $96 \%$, one can see that a higher muon yield can be obtained for a two-slab design at 35 degrees and $30 \mathrm{~mm}\left(15.5 \times 10^{-9} \mu / p\right)$ and a three-slab design at 40 degrees and $20 \mathrm{~mm}\left(12.2 \times 10^{-9} \mu / p\right)$. The surface muon yield doubles for the two-slab design case. It should be noted, however, that our simulations are based upon the existing ISIS muon beam line optics. Substantially higher muon beam intensities could be achieved if the optics were optimized specifically for the multiple slab geometries.

GEANT4 simulations were performed initially to investigate the proton energy dependence of the surface muon production for the current ISIS target parameters, indicating that $\approx 500 \mathrm{MeV}$ is the optimal energy for the ISIS target material and geometry [20]. Using the optimal target design described above, namely two slabs placed at $30 \mathrm{~mm}$ separation with an orientation angle of 35 degrees to the incident proton beam, the surface muon production shows an increase of $38 \%$ for the optimal energy of $500 \mathrm{MeV}$ [Fig. 9(b)].

\section{CONCLUSION}

Possible designs for a stand alone muon target for MuSR studies of condensed matter science are discussed in this paper. Using the ISIS target as a reference material and geometry optimizations are performed in this paper. Performances of materials like graphite, beryllium, and nickel are studied. The best candidate for a stand alone target is nickel. An increase in the surface muon yield is obtained by using a set of two and three slabs of the same total thickness as the initial target. A further increase can be obtained by varying the orientation angle made with the initial proton beam. An implication of the optimized solution for ISIS is discussed next. For ISIS where the proton transmission is an important factor that must be taken into account, the best material performance was found to be graphite. A higher increase in surface muons is obtained for a two-slab geometry at a separation distance of $30 \mathrm{~mm}$ and an orientation angle of 35 degrees.
Implementing in simulations the optimized solutions for the ISIS target, at the optimum beam energy of $500 \mathrm{MeV}$, it was found that an increase of $38 \%$ can be obtained with respect to the current ISIS parameters.

[1] A. Yaouanc and P. Dalmas de Réotier, Muon Spin Rotation, Relaxation and Resonance (Oxford University Press, Oxford, 2011)

[2] R. Heffner and K. Nagamine, Special issue on MuSR: Muon Spin Rotation, Relaxation or Resonance [J. Phys. Condens. Matter 16, 40 (2004)].

[3] S. L. Lee, S. H. Kilcoyne, and R. Cywinski, Muon Science (Muons in Physics, Chemistry and Materials) (Institute of Physics Publishing, Bristol, 1999).

[4] S. J. Blundell, Contemp. Phys. 40, 175 (1999).

[5] M. Burrard-Lucas et al., Nat. Mater. 12, 15 (2012).

[6] A. B. Mostert, B. J. Powell, F. L. Pratt, G. R. Hanson, T. Sarna, I. R. Gentle, and P. Meredith, Proc. Natl. Acad. Sci. U.S.A. 109, 8943 (2012).

[7] P. Dalmas de Réotier, A. Yaouanc, Y. Chapuis, S. H. Curnoe, B. Grenier, E. Ressouche, C. Marin, J. Lago, C. Baines, and S. R. Giblin, Phys. Rev. B 86, 104424 (2012).

[8] V. K. Anand, D. T. Adroja, and A. D. Hillier, Phys. Rev. B 85, 014418 (2012).

[9] A. Singh, E. A. Davis, E. Mytilineou, and M. Kay, J. Non-Cryst. Solids 198-200, 692 (1996).

[10] NMI3/CONFORM Workshop (Cockcroft Institute, Daresbury, 2008).

[11] F. Foroughi, E. Morenzoni, T. Prokscha, M. Daum, K. Deiters, D. George, D. Herlach, C. Petitjean, D. Renker, and V. Vrankovic, Hyperfine Interact. 138, 483 (2001).

[12] J. L. Beveridge, J. Doornbos, and D. M. Garner, Hyperfine Interact. 32, 907 (1986).

[13] G. H. Eaton, The Muon Beamline at ISIS, Rutherford Appleton Laboratory, 1994.

[14] Y. Miyake et al., Nucl. Phys. B, Proc. Suppl. 149, 393 (2005).

[15] E. Cartlidge, Phys. World 19, 13 (2006).

[16] GEANT4-version 4.9.3.p02, CERN [http://geant4.cern.ch].

[17] A. Ribon, J. Apostolakis, A. Dotti, G. Folger, V. Ivanchenko, M. Kosov, V. Uzhinsky, D. H. Wright, Report No. CERN-LCGAPP-2010-02, 2010.

[18] G. Folger, V. N. Ivanchenko, and J. P. Wellisch, Eur. Phys. J. A 21, 407 (2004).

[19] A. Heikkinen, P. Kaitaniemi, and A. Boudard, J. Phys. Conf. Ser. 119, 032024 (2008).

[20] A. Bungau, R. Cywinski, C. Bungau, P. King, and J. Lord, Phys. Rev. ST Accel. Beams 16, 014701 (2013).

[21] M. Kelsey, SLAC (private communication).

[22] D. R. F. Cochran, P. N. Dean, P. A. M. Gram, E. A. Knapp, E. R. Martin, D. E. Nagle, R. B. Perkins, W. J. Shlaer, H. A. Thiessen, and E. D. Theriot, Phys. Rev. D 6, 3085 (1972).

[23] Y. Miyake et al., Physica B (Amsterdam) 326, 255 (2003). 